\title{
Axiología y ciencia bibliotecológica: los valores en el mundo de la información documental
}

\author{
Miguel Ángel Rendón Rojas \\ Centro Universitario de Investigaciones Bibliotecológicas \\ de la UNAM, 04510, México D.F., Tel: 56-23-03-41 \\ E-mail: marr@servidor.unam.mx
}

\section{RESUMEN}

Se argumenta la posibilidad y necesidad de un estudio axiológico como parte de la fundamentación de la bibliotecología. Partiendo de que la axiología se desprende de la ontología, se concluye que el valor bibliotecológico surge y se fundamenta en el ser bibliotecológico que a su vez descansa en el ser del ser humano, por lo que se infiere la necesidad de recobrar el sujeto como parte central en el conocimiento bibliotecológico. La técnica y la tecnología son valores mediáticos, siendo el valor principal el propiciar el desarrollo del ser del hombre como ser informacional. Palabras Clave: Epistemología; Axiología; Valor; Bibliotecología.

\section{AXIOLOGY AND LIBRARY SCIENCE: VALUESIN THE WORLD OF DOCUMENTARY INFORMATION}

\author{
Miguel Ángel RENDÓN-Rojas
}

\begin{abstract}
The need and the possibility of an axiological study as part of the foundations of library science is argued. Stating as the point of departure that axiology issues from ontology, it is concluded that the library science value arises and is founded on the library science being, which in turn leans upon the being of the living creature. Therefore the need to recover the human subject as a central part of the library science knowledge, is inferred. Technique and technology are mediatic values, but the main value is to propitiate the development of the human being as an informational being. Keywords: Epistemolgy; Axiology; Value; Librarianship.
\end{abstract}

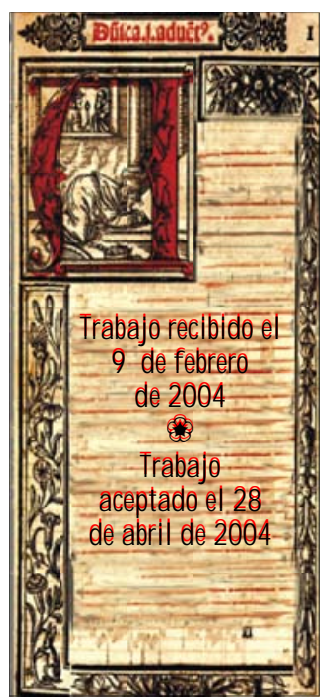


$\mathbf{E}$ 1 presente trabajo tiene como objeto analizar la problemática axiológica dentro del campo bibliotecológico y se enmarca dentro del programa propuesto por nosotros para la fundamentación de la ciencia bibliotecológica. ${ }^{1}$

Se tomará a la deniabiblidtedóġacomo la disciplina que tiene por objeto de estudio el sistema de información documental, el cual se analiza desde el punto de vista de la dinámica de los elementos que lo integran (información, documento, institución informativa documental, usuario, profesional de la información documental y actividades que realiza) para satisfacernecesidades de información documental del usuario. ${ }^{2}$

Por su parte la axidoǵaes una disciplina filosófica, por lo que es necesario determinar lo que se entiende por filosofía. A grandes rasgos, consideramos a la filosofía como un proceso inquisitorio a la realidad, tomada ésta como totalidad, tratando de responder a preguntas últimas tales como ¿qué es esa realidad, qué la identifica como tal y la diferencia de otras? ¿cuál es el material que conforma esa realidad y cómo es su estructura? ¿cuál es su origen, de dónde proviene y quién es su creador? ¿por qué y para qué existe? Al mismo tiempo la filosofía es el conjunto de respuestas que se obtienen de esa búsqueda y que están reflejadas en cuerpos de ideas, ya sean doctrinas sistemáticas (Aristóteles, Tomás de Aquino, Kant, Hegel, etcétera) u obras dispersas (Kierkegaard, Sartre). Lo característico del conocimiento filosófico es que se realiza con ayuda de la reflexión racional y un aparato teórico propio que incluye conceptos, juicios, raciocinios ymétodos específicos.

La axiología, también partiendo de una definición etimológica, proviene de la palabra griega $\alpha \xi 10 \sigma$ (axios) (valor) y $\lambda$ o $\gamma 0 \sigma$ (logos) (pensamiento, estudio, ley), lo que literalmente denota a la ciencia de los valores. Esa definición etimológica es deficiente, ya que en este caso se puede abordar dicho estudio desde el punto de vista psicológico, sociológico, antropológico, etcétera, sin que necesariamente ese análisis sea la búsqueda dela naturaleza, principios, fuentes y límites delos valores.

Así pues se tomará a la axiología como la disciplina filosófica que se pregunta por la naturaleza, principios, fuentes y límites de los valores ¿son éstos una realidad objetiva o subjetiva? Esto es ¿tienen las cosas valor porque las deseamos o las deseamos porque tienen valor? ¿Por qué existe la diversidad de opiniones que se manifiesta incluso en el proverbio latino degustibusnondsputandum ¿cuál es el criterio, la capacidad para captar el valor? ¿existe una jerarquía de valores?

A la pregunta qué son los valores se le pueden encontrar distintas respuestas. La primera de ellas es que los valores son formas o modos de la realidad irreductibles a otros; son una especie de cualidades especiales. Una segunda respuesta es que son

1 Miguel Ángel Rendón Rojas. "Las tareas de la fundamentación de la bibliotecología”, en Inestiga dónBiblicteedógica No 17, V. 8. pp. 4-9. México: CUIB, UNAM, 1994.

2 Miguel Ángel Rendón Rojas. Basesteénicasyfilosóficasdelabiblideedoǵa México: CUIB, UNAM, 1998. p. 135. 
producto de valoraciones humanas y por lo tanto son subjetivos y relativos. Una tercera respuesta expresa que los valores subsisten independientemente de las valoraciones y son los que hacen posible los juicios de valor; es una especie de platonismo axiológico, aunque se recalca que no es un mundo de "seres" sino de valores, y el valorno tiene como propiedad ontológica el ser sino el valer.

Según nuestro punto de vista, el valor es una relación del sujeto ante la cosa deseada. Los tres elementos resaltados son los que le dan razón de ser a la esfera axiológica, si desaparece uno de ellos se obscurece la comprensión del fenómeno valorativo. Actualmente por ejemplo, con el instrumentalismo, la tecnología pretende hacer a un lado al sujeto, y consecuentemente desaparecen los valores y se cae en lo impersonal, las máquinas crean información en sí, por sí y para sí. Los sujetos sólo observan y pretenden adaptarse, ir detrás de esa objetividad pura. El "truco" es descubrir el fetichismo de ese fenómeno, las máquinas que pretender serinteligentes eincluso "dialogar", no son más que un espejo que refleja lo que el hombre ha hecho, por lo que dicho diálogo es en realidad un monólogo.

Como fundamento y guía de análisis adoptamos lo que hemos denominado Realis modialéticoy que a nuestro juicio evita el peligro de caer en el relativismo, irracionalismo, solipsismo y dogmatismo. Las tesis principales del Realismo dialéctico consisten en primer lugar en adoptar una posición ontológica realista, esto es, en reconocerque el reino del ser es anterior a la actividad, al conocer, al valorar, y al dar sentido del ser humano.

La segunda tesis del Realismo dialéctico, consistente en reconocer que el ser es dialéctico, es universal infinito e histórico finito y, por consecuencia, para su estudio es necesario emplear la dialéctica como método central de análisis. Este método supone ver el fenómeno estudiado como una tensión de elementos contrarios, como una coexistencia en la realidad de extremos opuestos que aunque se excluyen mutuamente en una relación, se necesitan recíprocamente en otra. Esa coexistencia no es una mezcla amorfa en la que cada uno de los opuestos pierde su identidad, ni una sobreposición ecléctica de ellos, sino un sistema con individualidad propia y que al mismo tiempo posee elementos que a su vez conservan su individualidad, elementos que son a la vez absolutos y relativos. Esa visión dialéctica nos permite movernos libremente sin absolutizar pero tampoco sin menospreciar los opuestos de contrarios, así por ejemplo, reconocer la universalidad y la historicidad, la objetividad de los valores y la subjetividad valorativa, la construcción de sentidos y la no-subjetividad pura de sentidos; etcétera.

\section{NECESIDAD Y POSIBILIDAD DE UN ANÁLISIS SEMEJANTE}

A primera vista es posible cuestionar qué tan válido teóricamente es realizar un estudio axiológico dentro de la ciencia bibliotecológica. Se puede objetar que una disciplina no posee una relación inmediata con la ética y la axiología; por lo que si se desea profundizar en un estudio semejante, que se encuentra ligado a la esfera práctica, 
entonces se debe tener en cuenta no a la disciplina como tal, sino a la profesión que emana de esa disciplina y a los profesionales que ejercen talprofesión.

En efecto, podemos considerar a una teoría como un conjunto de enunciados, que en primer lugar, desde un punto de vista sintáctico, poseen una relación entre ellos y una estructura lógica que permite realizar inferencias de unos enunciados a otros; y que, en segundo lugar, desde un punto de vista semántico, se refieren a un estado de cosas en el mundo: describen y explican la presencia, las propiedades y las relaciones, determinadas por leyes o regularidades, de ciertos fenómenos reales en un mundo exterior al sujeto creador de la teoría; es decir, los enunciados son, o por lo menos tienden a ser, objetivos. De la misma manera, existen en bibliotecología enunciados que denotan "el frío estado de cosas" que suceden objetivamente, esto es, existen enunciados verdaderos independientemente de los valores o deseos del sujeto. Por ejemplo, en un sistema de clasificación bibliotecológico se enuncian relaciones sintácticas y semánticas objetivas entre los elementos de ese sistema, cosa que sucede en todo sistema semiótico no-contradictorio; 3 así también, entre las subclases de ese sistema se cumplen las relaciones que se formulan en la teoría intuitiva de conjuntos. ${ }^{4}$

Por consiguiente, de acuerdo con esta forma de razonar, podemos afirmar que el sujeto no pone nada de su parte para que dichos enunciados se cumplan. Es verdad que posteriormente, gracias a la verdad de la teoría, es posible predecir y controlar la realidad, lo que conlleva a transformarla y manipularla; pero la teoría en sí no prescribe ninguna norma acerca de la teleología de la actividad transformadora. Los fines y valores que orientan esa actividad teleológica están insertos en el sujeto y no en el objeto. El desear hablar sobre fines y valores es desear introducir subjetivismo en la disciplina en detrimento del objetivismo, es buscar que la teoría cumpla con una función esencialmente a ajena a ella: una función valorativa.

3 Una relación semántica clásica es la relación de denotación. Dado un símbolo $\mathrm{X}$ de un lenguaje, dicho símbolo tiene una relación con un objeto $\alpha$, tal que $\mathrm{X}$ nombra -denota, refiere- a $\alpha$. (En un lenguaje cerrado, el objeto $\alpha$ no debe pertenecer al mismo lenguaje.) Esta misma relación se da en la teoría bibliotecológica, por ejemplo, en un sistema bibliotecológico de clasificación sabemos que existen símbolos que denotan clases o los lenguajes documentales, que aunque comúnmente se dice son "representación del conocimiento", en sí son sistemas semióticos que "denotan", no sólo representan, conocimiento.

4 Por ejemplo, dados $\mathbf{U}$ que es un conjunto universal, $\mathrm{A}, \mathrm{B}$ y $\mathrm{C}$ que son conjuntos dentro de ese universo, Xque es un objeto, $\in$ símbolo de pertenencia de un elemento a un conjunto; $\notin$ símbolo de no pertenencia de un elemento a un conjunto; $\subset$ símbolo de ser subconjunto; $\bigcap$ símbolo para la intersección entre conjuntos; $\bigcup$ símbolo de unión entre conjuntos, entonces tenemos: no es posible que $\mathrm{x} \in \mathrm{A}$ y $\mathrm{x} \notin \mathrm{A}$; si $\mathrm{x} \in \mathrm{U}$, entonces $\mathrm{x} \in \mathrm{A}$ o $\mathrm{X} \notin \mathrm{A}$; si $\mathrm{x} \in \mathrm{A}$ y $\mathrm{A} \subset \mathrm{B}$ entonces $\mathrm{X} \in \mathrm{B}$ si $\mathrm{A} \subset \mathrm{By} \mathrm{B} \subset \mathrm{C}$, entonces $\mathrm{A} \subset \mathrm{B} \mathrm{A} \cap(\mathrm{A} \cup \mathrm{B})=\mathrm{A} ;(\mathrm{A} \cap \mathrm{U})=\mathrm{A}$; si $\mathrm{A}$ y $\mathrm{B}$ son conjuntos que no tienen elementos comunes entonces $\mathrm{A} \cap \mathrm{B}=\varnothing ; \mathrm{si} \neg \mathrm{A}$ es el complemento de $\mathrm{A}$ y $\mathbf{U}$ es el conjunto universal del cual $\mathrm{A}$ es subconjunto entonces $\mathrm{A} \cup \neg \mathrm{A}=\mathbf{U}$; entre otras muchas otras relaciones. Ésta es la razón por la que los operadores booleanos pueden aplicarse en búsquedas automatizadas. 
Sin embargo, tal posición es la aceptación de una tesis, a nuestro juicio criticable: la exclusión de fines y valores del ámbito racional, identificando la ciencia positiva con lo racional, por lo que se deja fuera todo otro tipo de posible racionalidad: la razón práctica, la razón dialógica, la razón prudencial (phrónesis). La anterior tesis es una nota distintiva propia del pensamiento moderno-positivista, el cual únicamente acepta la razón instrumental (conocer objetivamente para transformar y controlar), racionalidad que se desentiende delos fines para centrarse solamente en los medios.

Ahora bien, si continuamos con esa línea de pensamiento, se presentan unas interrogantes que exigen dada su importancia, tener una respuesta. Al fin y al cabo el conocimiento y la transformación son dirigidos por un fin y por valores: ¿de dónde provienen esos fines y valores? En el caso particular de la bibliotecología ¿son el resultado de una actitud arbitraria de los dirigentes y de los profesionales que se dedican al servicio bibliotecario? Pero está claro que la demarcación de los objetivos para los que se desea crear una red de bibliotecas determina los pasos a seguir para realizarlo; el para qué determina el cómo. A hora bien, esos fines y valores no pueden descansar en una esfera exterior de la disciplina sino en su interior; no se puede estar supeditado a que el Ministro de Educación en un momento dado sea un Vasconcelos y entonces todos trabajen para llevar los libros al pueblo, pero en otro momento sea un ingeniero en computación y entonces todos los esfuerzos se encauzan hacia la tecnología de la información, y en un tercer momento sea un fanático fundamentalista que ordene la destrucción de documentos no acordes con su ideología y todos se dediquen, con "los mejores medios", a cumplircon la tarea en turno.

La bibliotecología en su desarrollo histórico siempre ha estado ligada a la actividad práctica, y ya Husserl al analizar las disciplinas prácticas, llegó a la conclusión de que dentro de ellas se encuentra un sistema de valores, y que, más aún, ese sistema está jerarquizado, por lo que existe una norma principal con base en la cual se juzga el valor práctico de las acciones normadas por la disciplina práctica. ${ }^{5}$ El reto teórico es descubrir, delimitar y analizar cuál es esa norma y por quélo es. De acuerdo con Husserl, las reglas de la disciplina práctica expresan lo que ésta debe ser, señalan una exigencia para realizar cierta actividad de determinada manera y con ciertas condiciones, por lo que si se respeta esa exigencia, se alcanza un fin que se considera positivo. La tesis que defendemos es que el concepto de "bueno" (positivo) o "malo" (negativo) según los cuales se dividen los fines, no puede radicar en una decisión arbitraria y convencionalismos éticos, sino que tiene que hacerlo en una relación axiológica fundamentada en la ontología: el valor surge por el ser, se fundamenta en el ser, aunque al mismo tiempo recrea el ser; lo que en el campo bibliotecológico significa que el valor bibliotecológico surge y se fundamenta en el ser bibliotecológico y recrea el ser bibliotecológico, elcual a suvez se desprende del serdel serhumano.

5 E. Husserl. Inestigadones lógicas 1. Madrid: Alianza Editorial, 1985. pp. 60-65. 
La tesis instrumentalista-positivista no trabaja en esta esfera porque se considera a la bibliotecología como una ciencia del espíritu, en donde, con terminología de von Wright, ${ }^{6}$ la función epistemológica es fundamentalmente teleológica; es decir, el resultado del conocimiento no es explicar la realidad, sino comprenderla, encontrarle su sentido, el cual está dado en su intencionalidad; de esta manera, los fines, basados en valores, ocupan unlugar centralen el sistema de la disciplina.

¿Por qué, volviendo al ejemplo de los dirigentes del Ministerio de Educación, juzgamos como buen bibliotecario al que, en el caso de que el dirigente sea un "humanista"; pese a las indicaciones de los que tienen el poder, no se limita a cumplir con la política de crear bibliotecas públicas, sino que se preocupa también por la aplicación de la tecnología hasta donde sea posible en las bibliotecas; o, por el otro lado, en el caso de que el dirigente sea un "tecnólogo", no se ajusta únicamente a la automatización de las bibliotecas y se preocupa porque el libro llegue a la mayor parte posible de la población? ¿Por qué algunos bibliotecarios consideraron no aplicable el mandato de la autoridad -llámese Tlacaelel, Inquisición, Nacional-socialismo, Pol-Pot, democracia popular, o poder soviético-de destruir los libros? No es posible que esto haya sido con base en su criterio personal, puesto que ni son los grupos de poder, ni los "buenos" bibliotecarios quienes establecen las normas y los fines a seguir; sino que más bien se tata de normas y fines nacidos dentro de la visión teórica de la disciplina. La investigación de esos fines, para que sea científica, tiene que ser objetiva, (a pesar del subjetivismo que representa el hecho de que sean individuos concretos los "sustratos" de esos fines).

Por otro lado, al mismo tiempo que la axiología está inscrita dentro de la disciplina, se observa una influencia de la axiología en la disciplina; la primera condiciona el desarrollo de la segunda. Así por ejemplo, si en medicina se determina que la eutanasia es aceptable y deseable, entonces algunas de las investigaciones médicas se encauzarán a descubrir nuevos métodos para aplicar la eutanasia; o si se acepta que la manipulación es un valor positivo para la educación, entonces en la pedagogía se buscarán formas efectivas para manipular a los alumnos. De esta manera observamos que la aplicación práctica descansa en una teoría y toda la investigación es motivada por un fin aceptado en un principio.

En ocasiones se ha escuchado la preocupación de la comunidad bibliotecológica por el hecho de que ha querido adaptarse acríticamente a la tecnología, sin embargo debido a la incesante y rápida evolución de los artefactos tecnológicos se percibe que la bibliotecología siempre va detrás de los cambios en el área tecnológica, y cuando alcanza a uno de ellos y finalmente logra asimilarlo, se da cuenta de que ya es obsoleto, de que existen otros avances más sofisticados; o simplemente se descubre que ese logro tecnológico no era necesario para los fines que persigue la disciplina en su faceta práctica. Para evitar lo anterior es necesario detenerse a pensar ¿qué queremos? y ¿por qué y para quélo queremos? Y, consecuentemente, ¿hacia dónde se desea llegar?

6 George Henrik Von Wright. Explicacónycamprensión Madrid: Alianza, 1979. 
¿qué fin o fines se persiguen en la actividad informativa teórica (bibliotecológica) y práctica (bibliotecaria)? ¿Por qué y para qué deseamos ese fin? ¿Qué valores le dan fundamento a esa actividad? y ¿Qué fundamento tienen esos valores que determinan los fines?

Es decir, dentro de la comunidad epistémica y profesional del área de la información se percibe la necesidad de reflexionar sobre fines y valores, pero, obviamente, no para caer en homilías y buenos deseos, o constatar con tono pesimista lo que debería ser, pero que no es; sino para realizar una reflexión sistemática, racional y justificada; esto es, una investigación teórica que le proporcione ciertas coordenadas, no sólo a la actividad bibliotecaria, sino a la actividad bibliotecológica. Los resultados que se obtengan serán de mucho valor en cuanto que se tendrá un eje rector que nos podrá orientar en el torbellino de cambios, y nos ayudará a valorar las tradiciones y las innovaciones, el sery el hacer dentro del campoinformativo.

Si se logra cierta claridad sobre este punto, ya no se irá detrás de modas, con "sentimientos de culpa por no actualizarse", sino que se tendrá un criterio para aceptar lo que convenga al objetivo de la disciplina, ya sea algo novedoso o tradicional, se revalorizará el ser del profesional de la información, y se poseerá una justificación racional delCódigo de Ética.

Tales planteamientos no son preguntas retóricas, sino interrogantes cuyas respuestas nos pueden conducir teórica y prácticamente hacia caminos muy distintos. Una consecuencia sería si la respuesta es que el fundamento que rige los fines es "la mano invisible" del mercado, como propone el neoliberalismo; otra, sería si la respuesta es que son los grupos elitistas los que determinan los fines y valores, y otra distinta sería si la respuesta es que el valor no está en lo mercantil, ni en el poder, sino en elSerdel hombre.

Consecuentemente esto nos llevará a preguntarnos a su vez por el concepto de Hombre y Sociedad desde el punto de vista bibliotecológico, así como por la naturaleza del bibliotecario dentro de la sociedad ¿cuál es su función social? ¿quéle compete y qué no le compete? ¿cuáles valores rigen su actividad y porqué?

En este trabajo realizaremos una reflexión sobre aquellos valores mediáticos y finales que tienen influencia en el campo bibliotecológico. El punto de partida y referencia de estas reflexiones es situar a la ciencia bibliotecológica como ciencia del espíritu y consecuentemente como disciplina que cae dentro de los límites "subjetivos" culturales. La subjetividad relativa (individual o social) se supera gracias a la intersubjetividad que descansa en el reino del ser y que se descubre en el juego y en el diálogo. De ahí que los fines y valores de la bibliotecología como disciplina tengan a su vez la referencia ontológica de la intersubjetividad en el juego y en el diálogo y no en la manipulación con objetos (técnica, tecnología), ni en el monólogo de un Sujeto que "sabe" lo que se debe hacer y conocer (primacía de la política o economía). Pasemos a examinar con detalle una relación mediática en elámbito bibliotecológico. 


\section{LA TÉCNICA Y LA TE CN OLOGÍA COMO VALORES ME DIÁTICOS DENT RO DE LA BIBLIOTECOLOGÍA}

Uno de los fenómenos al que se enfrenta la investigación bibliotecológica es la técnica y la tecnología, las cuales en ocasiones son tomadas como males necesarios que se deben soportar porque no se pueden evitar, o, en otras, se las concibe como solución universal para todos los problemas. ¿Qué son y para qué sirven estos dos elementos? ¿Cuál es su contenido axiológico dentro del proyecto existencial del ser humano dentro delcontexto bibliotecológico?

Los griegos utilizaron el término $\tau \varepsilon \chi \nu \eta$ (téhnẹ para denotar una actividad que sigue determinadas reglas para producir una realidad artificial. Aristóteles va más allá y distingue cinco capacidades para conocer $y$, por consecuencia, cinco tipos de conocimiento: la experiencia que produce el conocimiento cotidiano; la técnica que proporciona el conocimiento técnico (téchne; la phrónesis(prudencia) que da el conocimiento práctico en las acciones; el razonamiento que produce la ciencia (episteme) y la intuición que proporciona el conocimiento de las esencias.

De esta manera, la técnica se concibe como un modo de asimilar y transformar la realidad, se trata de un "saber hacer" de acuerdo con reglas que se deben seguir para obtener el producto deseado; es superior a la experiencia simple porque ésta únicamente "da cuenta de un estado de cosas" sin la intención de querer influir en ellas, mientras que la técnica busca influir en la realidad mediante una actividad regida por reglas para producir o transformar un objeto. Al mismo tiempo la técnica es inferior a la ciencia, ya que ésta se preocupa por investigar las causas que rigen el devenir de los fenómenos, mientras quela técnica no puede dar explicación del por qué de las reglas que se utilizan.

Es en la Edad Moderna cuando, con su paradigma de poder, se exalta la técnica como valor último de la capacidad humana, e incluso se reduce la ciencia a ese fin. Podemos recordar la posición de F. Bacon, quien afirmara que "el conocimiento es poder". En la actualidad, nuestra sociedad contemporánea, como producto del proyecto de la modernidad no se ha apartado de esa visión, y podemos observar que en la asíllamada sociedad de la información se llega aún más lejos, sustituyendo el conocimiento porla simple información, afirmando la tesis de que la "información es poder". 7

Por su parte, concebimos la tecnología también desde el marco aristotélico, aunque con una nueva interpretación de los tipos de conocimiento. Así pues, se agrega un nuevo tipo de conocimiento: la tecnología, que es independiente y no la simple unión de la episteme con la téchne, es un conocimiento universal-concreto, necesario contingen$t^{8}$, porque por un lado parte de una necesidad concreta para transformar la realidad y,

7 Es cierto que en ocasiones se habla de Sociedaddd conoimiento pero en este caso se trata más bien de un conocimiento tecnológico. Cf Rendón Rojas, M. A. "Sociedad del conocimiento", en Lasode daddd conoimiento México: UNAM/CUIB. 2000. pp. 50-57. pp. 54-55.

8 Cosa que para Aristóteles sería impensable. Para Aristóteles fue obvio que el hacer no era un fin en sí mismo y que estaba subordinado a varias posibles concepciones del bien, como con los sistemas políticos con los cuales estas concepciones están vinculadas. Cfr. Ética a Nicómaco 1094a. 
como consecuencia de su aplicación, se producen o transforman objetos individuales; pero al mismo tiempo, las reglas que rigen ese accionar son producto de una investigación científica, por lo que es posible indicar las leyes y causas por las cuales esas reglas son eficaces. Es característico de este tipo de conocimiento, crear modelos, los cuales son construcciones ideales que reproducen la estructura y funcionamiento del fragmento de la realidad que representan. De esta manera, la modelación de los fenómenos y procesos, basada en la identificación, la simbolización lógica y matemática de elementos y relaciones, la reconstrucción ideal de todo el proceso o fenómeno, y le descubrimiento de leyes y regularidades, permite crear ya no sólo objetos de manera inmediata, sino incluso objetos de manera mediata, a través de artefactos que a su vez son capaces de reproducir objetos, fenómenos o procesos. Ese primer artefacto es el objeto tecnológico, producto del conocimiento tecnológico, pero no es la tecnología. Ésta es el conocimiento, aquél es el producto.

Por otra parte, consideramos que se deben diferenciar técnica y tecnología con base en el tipo de conocimiento utilizado para transformar la realidad. Mientras que la primera no puede justificar, esto es, no está en condiciones de dar las razones del por qué de las reglas que se deben utilizar en el proceso de la acción, porque está unida a la empiria; la tecnología, por el contrario, es capaz de proporcionar la explicación teórica que fundamenta el correcto funcionamiento de la acción cuando se siguen las reglas. Así por ejemplo, aunque es posible hablar de cierta tecnología de la construcción en la antigua Mesopotamia, según nuestra visión, es mejor hablar de técnica de la construcción, porque aquellos pueblos no lograban explicar por qué el sol lograba convertir el barro en ladrillos con una consistencia más dura; del mismo modo, la utilización de la pólvora en juegos e incluso en armas de fuego, no puede ser considerada tecnología, sino hasta el momento en que se conocen los procesos químicos y físicosque permiten desencadenarlos procesos de explosión eimpacto.

Lo anterior, aplicado en el contexto de la ciencia bibliotecológica, significa que cuando se habla de técnica se debe entender aquella acción que actúa sobre la realidad para transformarla, pero que no puede fundamentar teóricamente las reglas que rigen esa acción. Si se logra identificar el momento teórico se pasa al nivel del logos lo cual implica que se realiza una investigación científica, por lo que se desarrolla como ciencia; ${ }^{\circ}$ o se desarrolla una investigación tecnológica, si es que se tiene como objetivo construir un objeto artificial, un artefacto. Así pues, la tecnología en ciencia bibliotecológica es el conocimiento que conduce a la creación de artefactos que posteriormente ocupan un lugar en el Sistema Informativo Documental, conocimiento que incluye elementos teóricos como fundamentos de la operación de dicho artefacto.

9 Cf. M. A. Rendón Rojas. Basesténicasyfilosóficasdelabiblidtedoǵa México: UNAM/CUIB, 1998. pp. 43-47. Donde se defiende la tesis derivada de las ideas de Husserl de que siempre existe un fundamento teórico sobre el que descansan las normas que rigen una actividad práctica. 
De esta manera, se debe tener cuidado al hablar de tecnología de la información o "las nuevas tecnologías de la información", ya que en ocasiones la intención directa de ese término tiene como significado los artefactos tecnológicos y no el tipo de conocimiento que los creó. Como ejemplo de artefactos tecnológicos tenemos diversos soportes que sirven para conservar información (discos, diskettes, CD, cassetes, videos, etcétera); los “lectores" o aparatos que sirven para mostrar el material codificado que se guarda en los soportes; las computadoras que se emplean para agilizar el flujo de la información, ya sea para guardar, organizar, transmitir información; las redes internas o Internet; los canales que se utilizan, etcétera.

Al mismo tiempo no podemos negar el hecho de que el mismo sistema de información documental y consecuentemente las relaciones que mantiene con otros sistemas, dependen de una intencionalidad y por consiguiente de fines y sentidos. Por lo anterior, afirmamos que el origen, el centro y el fin de la acción bibliotecológica tiene un elemento humano-social, de ahí que califiquemos a la ciencia bibliotecológica como ciencia del espíritu y consecuentemente a partir de eso surge la base axiológica.

Por lo tanto podemos ver la importancia del sujeto dentro de la ciencia bibliotecológica. Él crea el acontecimiento significativo: información, documento, necesidad de información, institución informativa documental, actividades para adquirir, conservar, organizar, transmitir información, etcétera. Él es quien con su diálogo con otros sujetos nos ayuda a comprender el acontecimiento significativo. Esos tres elementos son centrales: sujeto, diálogo y comprensión.

Sin embargo, en la actualidad, con la aparición de tecnologías cada vez más sofisticadas, parece que las máquinas creadas para funcionar dentro del sistema de información documental, después del impulso inicial que le confiere la primera causa, el ser humano que les infundió el espíritu (softharè en su cuerpo (hardmarê, empezaran a actuar de manera autónoma y como individuos, pero no como sujetos: crearan información, la procesaran, la transmitieran y en algunas ocasiones parecequela comunicarany el ser humano "dialogara” con sus creaciones.

Por definición, tomamos al diálogo como la interacción de dos sujetos que buscan llegar a un consenso justificado. Si en el proceso de interacción se suprime el sujeto, obviamente ya no hay diálogo. La máquina no es sujeto, no tiene mundo de vida, no tiene intenciones, no le interesa llegar a un consenso ni a la comprensión. Debido a lo anterior, podemos concluir que no se da el diálogo entre el sujeto y elobjeto.

Si recordamos el concepto de verdad de Heidegger como aleheia des-velación, des-cubrimiento del ser, ${ }^{10}$ entonces cuando la técnica es un modo de conocer, episte metechné no existe ningún problema porque ella ayuda a la producción de verdadero en lo bello. Sin embargo en la época moderna, con su complejidad, la técnica, como ya lo hemos visto, puede dejar de ser un modo de conocer y no ser más un medio para

10 Cf M. Heidegger. Elsæryd tiempa México: F.C.E., 1971.』44. Así como Heidegger, M. Ddl’aœsena ddlaveita Milano: Fratelli Rocca, 1952. 
la manifestación del ser, sino, por el contrario, traicionar a la aleheiay convertirse no en des-velación del ser sino en ocultamiento deél.

Sin embargo, consideramos que ese problema no es de la técnica en sí, sino del sujeto acrítico que se relaciona con ella y no la interpela. Utilizando la misma terminología de Heidegger, el problema es del hombre que tiene una existencia in-auténtica, que usa la tecnología porque se usa, pero nola comprende.

Así pues, después de esta reflexión podemos entender a la tecnología como vehículo que permite la transmisión de datos que posteriormente se convierten en información, no es el agente que con su accionar crea la información. La tecnología es importante para el flujo de la información, pero no el fin en sí mismo; no es valor en sí mismo. El sistema de comunicación se da entre sujetos y por lo tanto la tecnología y sus artefactos, como objetos, no son parte esencial de dicho sistema; sólo ocupan un lugar periférico.

Para calificar el funcionamiento de un sistema debe de existir un parámetro que permita emitir tales juicios valorativos.¿Cuál es ese parámetro en el sistema de información documental?

Consideramos que la respuesta es unívoca: es el hombre, no como sistema biológico, ni psíquico, sino como ser que es centro y creador de los sistemas y, en nuestro caso, del sistema de información documental. Todos los sistemas se circunscriben dentro del ser del hombre. Ese parámetro buscado que le da el fundamento a la axiología es el ser del hombre; un sistema económico, político, científico, educativo, jurídico y de información documental, son del y para el hombre. La misma existencia de diferentes tipos de sistemas muestra las diferentes facetas del ser del hombre que en ocasiones incluso han sido elevadas a características esenciales que definen todo el ser del hombre. Así por ejemplo, Aristóteles afirmó que el hombre es animal político, por lo que no es casualidad que exista un sistema político; en la edad media se dijo que el hombre es un animal racional, por lo que hay un sistema científico; en la edad moderna se toma al hombre como ser con razón estratégica que controla y transforma la realidad, por lo que existe el sistema tecnológico; Locke y Cassirer escribieron que el hombre es un ser simbólico y vemos que surge un sistema lingüístico; Marx define al hombre como un animal económico, de ahí que exista un sistema económico; algunos otros afirman que su característica esencial es ser un animal social, por lo que se tiene un sistema social. En el caso que nos interesa en la esfera bibliotecológica, vemos al hombre como serinformacional.

Cada sistema tiene su código por medio del cual interpreta la realidad, el de la ciencia es el de verdad y no verdad, lo legal/no-legal es el del sistema jurídico, el de esti$\mathrm{ma} /$ desestima es el del sistema ético, el de tener/no-tenerel deleconómico, etcétera.

El código del sistema de información documental no solamente es recuperar o no información, sino recuperarla en función de un sujeto que se acerca a ese sistema para satisfacer necesidades de información. Por lo tanto, consideramos que el auténtico código del mencionado sistema es satisfacernecesidades de información documental/ no-satisfacer necesidades de información documental, entendiendo 
que el satisfacer implica coadyuvar al desarrollo existencial del sujeto, y no solamente proporcionarle la información que cree necesitar, sino incluso la que no sospechaba que necesitaba.

Ahora bien, el código de cada sistema con la presencia del sujeto puede ser visto como un valor: la verdad, la legalidad, la estima, la propiedad, la satisfacción de necesidades de información documental. Esto es una consecuencia muy importante porque entonces aparece el elemento axiológico en los sistemas. Esa axiología es precisamente la que nos va a permitir valorar el funcionamiento de los sistemas y en el caso concreto del sistema de información documental, nos dará una directriz para su investigación y para la docencia.

\section{RECOBRANDO AL SUJETO}

De todo lo hasta ahora dicho, se puede observar que la dirección hacia la que apunta nuestra propuesta consiste en retomar el ser del hombre como centro y causa del sistema de información documental, y por lo tanto como fundamento de la axiología que de aquí se desprende. La forma del ser del hombre condiciona la axiología que guía la dinámica de dicho sistema. Ahora la pregunta, planteada ya anteriormente, es ¿qué clase de ser es el que descubrimos en la persona humana? ${ }^{11}$ En otro trabajo hemos mencionado, siguiendo a Heidegger, la historicidad del ser del hombre, ser que se desarrolla en el tiempo y en el mundo, lo cual exige la existencia de un pasado capaz de ser conservado y transmitido, así como de un proyecto, elaborado con base en ese pasado, para construir el porvenir; es decir, determina usar tradiciones y al mismo tiempo hacer tradiciones, lo que hace al ser humano un serque consume y produceinformación. ${ }^{12}$

A esa visión de ser histórico, agregaremos, apoyándonos en el pensamiento de E. Cassirer, la característica del ser humano como ser simbólico. El ser humano se encuentra en y enfrenta a un mundo externo a él, un ser en sí que se presenta ante el hombre, pero que se representa a través de símbolos, es mediatizado por ellos para poder ser asimilado por la conciencia humana. Sólo los símbolos tornan transparente la huella de lo otro, la realidad se torna fenómeno como representada y únicamente como representada, se podría agregar. Como lo expresa Cassirer: "El hombre no puede enfrentarse ya con la realidad de un modo inmediato; no puede verla, como si dijéramos, cara a cara [...] En lugar de tratar con las cosas mismas, en cierto sentido, conversa constantemente consigo mismo [...] no puede ver o conocer nada sino a través de la interposición de este medio artificial [las formas simbólicas]."13 La representación es la función básica de la conciencia trascendental y sólo mediante los mundos

11 Por supuesto que la pregunta por el ser del hombre se realiza no de una manera abstracta, general y universal, sino desde la perspectiva de la ciencia bibliotecológica.

12 Miguel Ángel, Rendón Rojas. Bases teénicas y filosóficas dela biblideedoǵa México: CUIB/UNAM, 1998. pp. 38-39.

13 Ernest Cassirer. Antropologáa filosófica México: FCE, 1999. pp. 47 y 48. 
de imágenes articulados en las formas simbólicas "descubrimos y nos hacemos de aquellos [siqq que llamamos 'realidad'."14

Gracias a esa modelación simbólica el ser humano no es absorbido por ese mundo infinito pura presencia, sino, por el contrario, tiene la posibilidad de vivir en el mundo como parte de él pero al mismo tiempo diferente y ocupando un lugar estratégico dentro de dicho mundo. Con base en esa representación es como el hombre actúa, vive, se relaciona con el mundo.

Por otra parte, si consideramos que los símbolos creados constituyen un sistema semiótico, y el lenguaje es el sistema semiótico por excelencia, podemos comprender la frase de Heidegger lapalabra... [Alenguaje] eslacasadd s $^{45}$ ya que el lenguaje se convierte en el mediador entre el hombre y el mundo. Todo, absolutamente todo-emociones, afectos, sentimientos, sensaciones, percepciones, creencias, ideas, etcéteraaparece en el lenguaje. De aquí también el famoso aforismo de Wittgenstein loslímites demilenguajesigificanloslímitesdemimunda ${ }^{16}$ El hombre se mueve en un mundo representado, incluso cuando se le presenta algo nuevo y desconocido lo tiene que representar para afrontarlo aunque sea llamándolo con un signo tipo X: la nada, el éter, algo, una cosa, etcétera.

Sin embargo el lenguaje entendido como instrumento de comunicación (oral o escrita) no agota los sistemas simbólicos. Para Cassirer, la ciencia, el arte, la religión, la filosofía, el mito, son sistemas simbólicos; en pocas palabras la cultura, como conjunto de esos sistemas simbólicos, es en sí un sistema de símbolos. El ser humano no es un ser natural, sino cultural, el mundo donde se encuentra es un mundo "para nosotros", no un mundo "en si". Al ser un mundo interpretado culturalmente es un mundo de sentidos, por lo que el hombre, al ser un ser simbólico, ${ }^{17}$ es también un ser creador de sentido. Esos sentidos en ocasiones se plasman en documentos, se representan en documentos, y para tener acceso al mundo a través del sentido se necesita teneracceso al documento.

Por otra parte, siguiendo la tradición hermenéutica, el ser del ser humano se caracteriza porque el hombre es un sujeto, lo que significa que es un ser dialógico y un ser social. Expliquemos esto último, la diferencia tradicional en el esquema del conocimiento es entre sujeto-objeto. Pero si analizamos ese esquema desde el punto de vista del objeto, descubrimos que éste es algo mudo, que está ahí presente sin decir nada, es el mundo de la naturaleza, una roca, una estrella, un átomo. Si el sujeto es lo que se contrapone al objeto, lo opuesto a él, y el objeto es algo mudo, entonces el sujeto es algo que habla, más bien, alguien que habla. El sujeto es un ser dialogante. Pero si preguntamos dialogante con quién, descubrimos que no puede ser con un objeto, porque sería anormal dialogar con una cosa que no escucha y no puede responder;

14 E. Cassirer. La filosofía delasfomassimbálicas México: FCE., 1971. p. 57.

15 M. Heidegger. Carta sdbred humanismo México: Ediciones Peña Hermanos, 1998. p. 65, 71.

16 L. Wittgenstein. Tradatuslógicofiloś́ico 5.6.

17 Ernest Cassirer. Antropoloǵa filosófica México: FCE, 1999. p 49. 
tampoco puede dialogar consigo mismo porque sería un monólogo solipsista que no conduce a ninguna parte más que a un narcisismo patológico, incluso el mismo lenguaje que utiliza para ese monólogo es patrimonio social, sacado y aprendido en comunidad. Por lo tanto, el gran descubrimiento de la filosofía hermenéutica es que el sujeto no surge de su oposición al objeto, sino que el sujeto necesita de otro sujeto para ser sujeto. Si no existe el otro, no existe el yo. Sólo en el diálogo y en la sociedad es donde aparece el sujeto.

\section{CONCLUSIONES}

Esas características ontológicas: historicidad, creador de sentido y subjetividad son las que soportan los valores que rigen la actividad del sistema informativo documental. Si se exige profesionalismo en la actividad, si se debe respetar la privacidad, evitar la censura, promover la capacitación, etcétera, es para desarrollar al sujeto, que crea y recrea sentidos, información en este caso; que se sirve de su sistema de información documental para existir, esto es, para hacerse y hacer historia; y que se actualiza como sujeto al dialogar con otros sujetos, productos de su devenir cultural. Y todo esto se debe tener presente no sólo para la actividad práctica; sino que el desarrollo teórico de la disciplina debe tomar en cuenta esas características para no dejarse arrastrar por la tecnología, la política, la economía, e incluso el mesianismo de tipo humanista o social-revolucionario.

\section{BIBLIOG RAFÍA CONSULTADA}

ARISTÓTELES. Ética aNić́maco Madrid: Rubio, 1931.

CASSIRER, Ernest. Lafilosafía delas fomas simbólicas México: FCE, 1971.

- -. Antropoloǵafilosófica México: FCE, 1999.

HEIDEGGER, Martin. Carta sdared humanisma México: Ediciones Peña Hermanos, 1998.

- -. Dell'a eserzaddlaverita Milano: Fratelli Rocca, 1952.

- - . El seryd tiempa México: FCE, 1971.

HUSSERL, Edmund. Investigaciones lógicas, 1. Madrid: Alianza Editorial, 1985.

RENDÓN ROJAS, Miguel Ángel. "Sociedad del conocimiento”, en Laso cieladdd conoimiento México: UNAM/CUIB. 2000. pp. 50-57.

- - "Las tareas de la fundamentación de la bibliotecología", en InvestigaaónBiblictedógica No 17, V. 8. pp. 4-9. México: CUIB, UNAM, 1994.

--. Basesteónicasyfilosóficasdelabiblideedoǵa México: CUIB, UNAM, 1998.

WITTGENSTEIN, Ludwig. Tradatuslóġófilosófica Madrid: Tecnos, 2002.

WRIGHT, George Henrik Von. Explicacónycamprensión Madrid : Alianza, 1979. 198 p. 


\section{BIBLIOGRAFÍA COMPLEMENTARIA}

Brown César, Javier. Elementos para una terńabiblicterana México: ENBA, 2000. 224 p.

Chubarian, O. S. Biblideedoǵageneral. La Habana: Editorial Científico Técnica, 1976.

Curras, E. Lainfomadónensusnuevosaspetos Madrid: Paraninfo, 1988. 307 p.

Gómez, Villalpando Armando. El djełodeestudiodelaCieniadelaInforma dón Tesis para obtener el grado de maestro en Ciencias de la Información. Guanajuato, Gto. 1990. 81 h.

Gorbea Portal, S. y E. Setien Quesada. "De la Bibliotecología al Sistema de Conocimientos científicos Bibliotecológico-Informativo", en Investiga dónBiblidtedóġa V. 8. N. 16. pp. 21-25. México: UNAM, CUIB, 1994.

Lafuente López, R. "La síntesis crítica del conocimiento bibliotecológico: su valor para la investigación bibliotecológica”, enInvestigacónbiblidteo lóġa V. 3. N. 6. p. 3-10. México: UNAM, CUIB, 1988.

- - " "La síntesis crítica del conocimiento bibliotecológico: su valor para la investigación bibliotecológica", enInvestigacónbiblideedógica V. 3. N. 6. p. 3-10. México: UNAM, CUIB, 1988.

López, Yepes José. Ladbamentacióncomodisciplina. Tenáaehistaria Navarra: Eunsa, 1995. 337 p.

Mikhailov. A. I. y otros. Fundamentosdelainformática La Habana: Nauka y Academia de Ciencias de Cuba. Instituto de Documentación en información científica y técnica. 1973. 2 v. 718 p.

Puntel Mostafa, Solange. "Enfoques paradigmaticos da bibliotecologia; unidade na diversidad ou diversidad na unidad", en Investigaciónbiblide đdóġa V. 10, N. 21, julio-diciembre 1996. pp. 18-21. México: UNAM, CUIB, 1996.

Saracevic, Tekfo. Introductiontoinfommationsaience New York: R. R. Bowker, 1970. $751 \mathrm{p}$.

Sequeira, Ortiz D. y Sequiera, Ortiz Z. La Biblidtedoǵa comociencia San José, Costa Rica: Publitex, 1988. 218 p.

Shera, J. Losfundamentosdela@uradónbibliđtedoóg்a México: UNAM/CUIB, 1990.520 p.

Vickery, Brian Campbell. Infomationsienceinthetheryandpractice London. Butterworths, 1987.384 p.

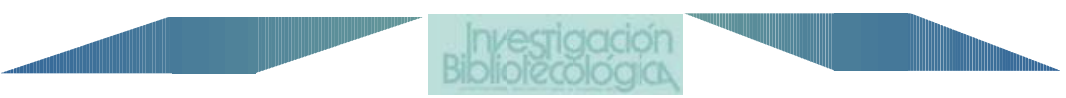

